\title{
DERMOSCOPIA DO LINFANGIOMA CIRCUNSCRITO NEVIFORME
}

\author{
Victoria Guiote Domínguez \\ Serviço Dermatologia do Hospital Santo André, Leiria-Pombal, Portugal
}

\begin{abstract}
RESUMO - O linfangioma circunscrito neviforme (LCN) é uma malformação linfática clinicamente caracterizada pela presença de múltiplas vesículas de conteúdo claro e/ou serohemático, agrupadas num "padrão herpetiforme" típico. Ocasionalmente, o LCN pode afetar a hipoderme, o músculo e/ou outros órgãos, o que dificulta enormemente a sua abordagem terapêutica. Apresentamos o caso clínico de um doente de 17 anos com LCN, encaminhado para à consulta de Dermatologia a fim de avaliar a adequação a terapêutica apropriada. Descreve-se o padrão dermatoscópico do LCN e os seus principais diagnósticos diferenciais.
\end{abstract}

PALAVRAS-CHAVE - Linfangioma; Dermatoscopia; Neoplasias da pele; Nevus.

\section{DERMOSCOPY OF LYMPHANGIOMA CIRCUMSCRIPTUM NEVIFORME - A CASE REPORT}

ABSTRACT - The Circumscriptum Neviform-like Lymphangioma(CNL) is a lymphatic malformation clinically characterized by the presence of multiple vesicules of a clear or a serum/hematic content, grouped in a typical "herpetiform-like pattern". Ocasionally, the CNL may affect the hypoderm, muscle and/or other organs, which may turn particularly difficult its therapeutics. We present a clinical case of a 17 year old patient with CNL referred to the Dermatology Department in order to be done a clinical evaluation, namely if the medication was the appropiate one. In this article we describe the dermatoscopic pattern of the CNL as well as the main differential diagnosis.

KEY-WORDS - Skin neoplasms; Lymphangioma, dermatoscopy; Nevus.

\section{Conflitos de interesse: Os autores declaram não possuir conflitos de interesse. \\ No conflicts of interest. \\ Suporte financeiro: O presente trabalho não foi suportado por nenhum subsídio ou bolsa. \\ No sponsorship or scholarship granted.}

Recebido/Received - Maio/May 2012; Aceite/Accepted - Junho/June 2012

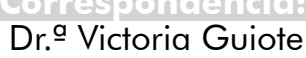

Serviço de Dermatologia

Hospital Santo André

Rua das Olhalvas- Pousos

2410-197, Leiria

Tel: + 351244817000

E-mail:viviguiote@gmail.com 


\section{Caso Clínico}

\section{INTRODUÇÃO}

O linfangioma circunscrito neviforme (LCN) é uma malformação linfática, pouco frequente, que corresponde a um hamartoma e que ocorre nos primeiros anos da infância.

Caracteriza-se tipicamente por múltiplas vesículas de conteúdo claro e/ou serohemático, agrupadas num "padrão herpetiforme" . Pode afetar a hipoderme, o músculo e/ou outros órgãos.

A sua etiologia parece estar relacionada com um aumento da pressão a nível dos vasos linfáticos da pele conectados a cisternas localizadas na hipoderme (hipótese de Whimster) ${ }^{2}$. Tais características fazem do LCN uma malformação com uma abordagem terapêutica difícil.

\section{CASO CLÍNICO}

Doente de 17 anos do sexo masculino referenciado à consulta de dermatologia para avaliação de uma lesão cutânea presente desde o nascimento, localizada na região lombar direita, de grandes dimensões e que desejava excisar.

Cerca de três anos antes, a lesão havia sido excisada parcialmente, tendo ocorrido recidiva local do total das áreas excisadas nos meses seguintes.

O exame objectivo revelou a presença de múltiplas vesículas, milimétricas, de conteúdo serohemático agrupadas segundo uma distribuição herpetiforme, sobre uma base eritematosa (Fig. 1).

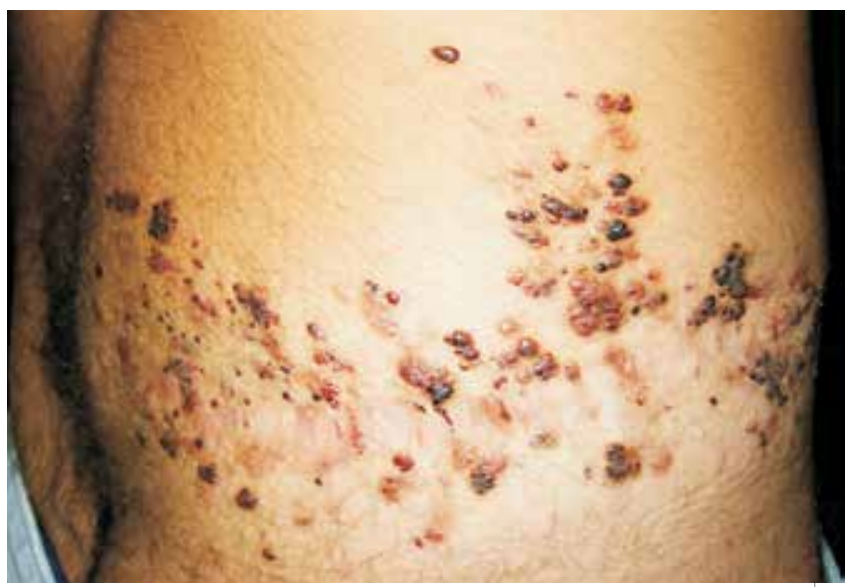

Fig. 1 - Múltiplas vesículas, de conteúdo serohemático agrupadas com distribuição herpetiforme, em base eritematosa.
A dermatoscopia mostrou múltiplas lacunas, algumas translúcidas e outras de conteúdo serohemático, de diferentes tonalidades, separadas por septos esbranquiçados (Figs. 2,3 e 4). Os referidos achados eram compatíveis com o diagnóstico de LCN, que se confirmou igualmente por uma biopsia incisional da lesão.

Com o objetivo de avaliar com mais pormenor a possível extensão em profundidade da lesão, solicitou-se uma RMN da região lombar direita e da pélvis que mostrou uma massa retroperitoneal, de contornos mal definidos e irregulares com envolvimento do músculo ilíaco direito e com desvio anterior do músculo psoas

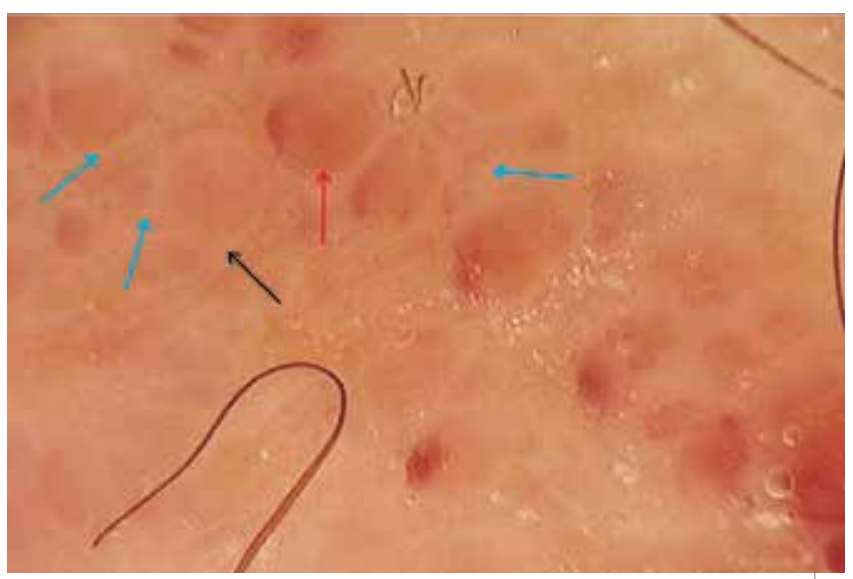

Fig. 2 - Dermatoscopia: múltiplas lacunas, algumas translúcidas (seta preta) e outras de conteúdo serohemático (seta vermelha), separadas por septos esbranquiçados (setas azuis).

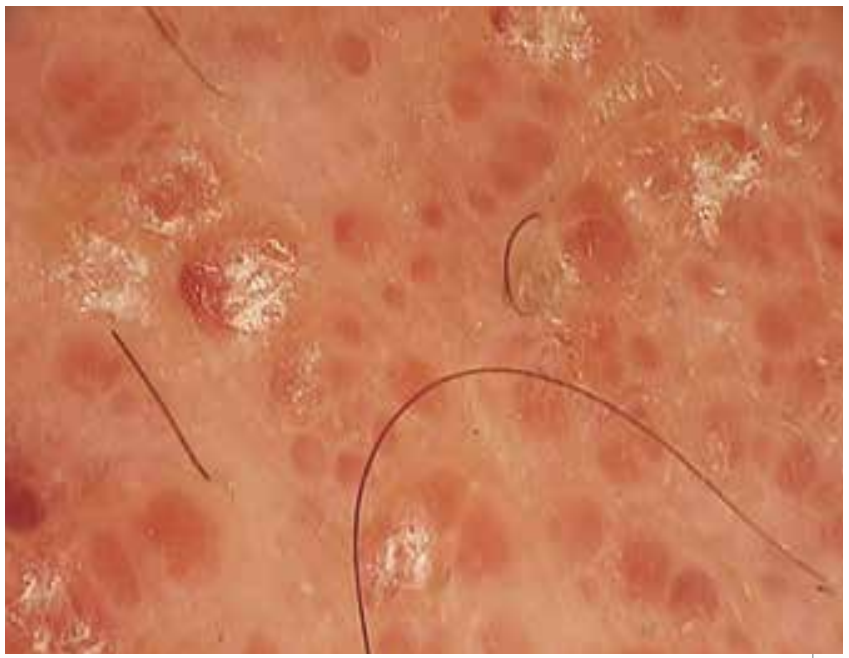

Fig. 3 - Dermatoscopia: os mesmos aspectos de Fig.2. 


\section{Caso Clínico}

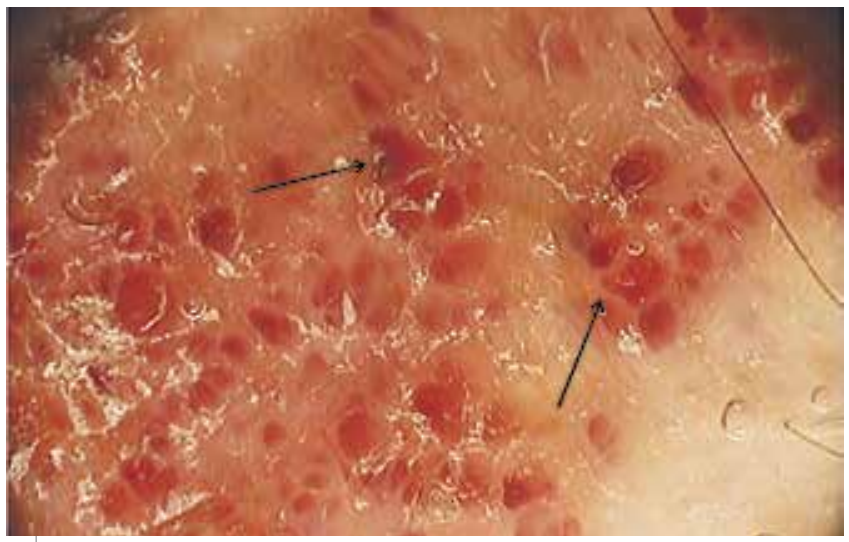

Fig 4 - Dermatoscopia: lacunas de conteúdo hemático escuro (setas).

homolateral, compatível com a existência de componente profundo pelo que não foi realizada qualquer intervenção cirúrgica.

Perante este caso clínico foi adotada uma atitude expectante, sendo o doente mantido em seguimento, clínico e dermatoscópico, semestralmente.

\section{DISCUSSÃO}

O LCN é uma malformação linfática de incidência desconhecida. Embora possa já ser diagnosticada in utero, na maioria dos casos, o diagnóstico é efectuado entre os 2-3 anos de idade ${ }^{3}$. Deve-se a um erro durante a morfogénese dos vasos linfáticos.

A sua etiologia está associada a um aumento da pressão ao nível dos vasos linfáticos da pele, os quais se encontrariam conectados a cisternas localizadas na hipoderme (hipótese de Whimster)2. Tal hipótese justificaria, a drenagem ocasional de um líquido seroso, que corresponde a linfa.

O LCN pode apresentar um componente profundo considerável, pelo que o seu tratamento pode tornar-se complicado, dada a elevada probabilidade de existir uma recidiva posterior, como no caso do nosso doente.

O diagnóstico de suspeita é clínico e deve ser confirmado pelo "gold standard" que continua a ser, sem dúvida, o exame histopatológico.

Contudo, a dermatoscopia representa uma ferramenta muito útil no diagnóstico desta patologia ${ }^{4}$, e caracteriza-se pela presença de múltiplas vesículas agrupadas, algumas translúcidas e outras com conteúdo serohemático, de diferentes tonalidades de vermelho, separadas por septos esbranquiçados.
Entre os diagnósticos dermatoscópicos diferenciais possíveis destacamos dois:

1. Angioma (Fig. 5): caracterizado pela presença de um padrão lacunar vermelho, de diferentes tons, que ocasionalmente pode apresentar uma área central de despigmentação branca cicatricial, histologicamente correspondente a fibrose. Tal característica é típica, por exemplo, dos hemangiomas senis fibrosados.

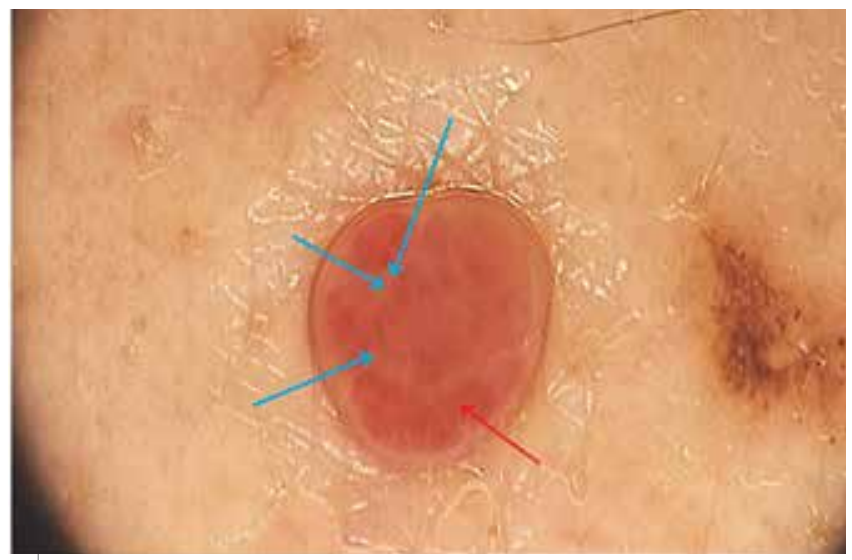

Fig 5 - Dermatoscopia de Angioma: padrão lacunar vermelho (seta vermelha), de diferentes tons, com área central de despigmentação branca cicatricial (setas azuis).

2. Angioqueratoma (Fig. 6): Apresenta lacunas mais escuras que o angioma e um véu esbranquiçado ${ }^{6-8}$.

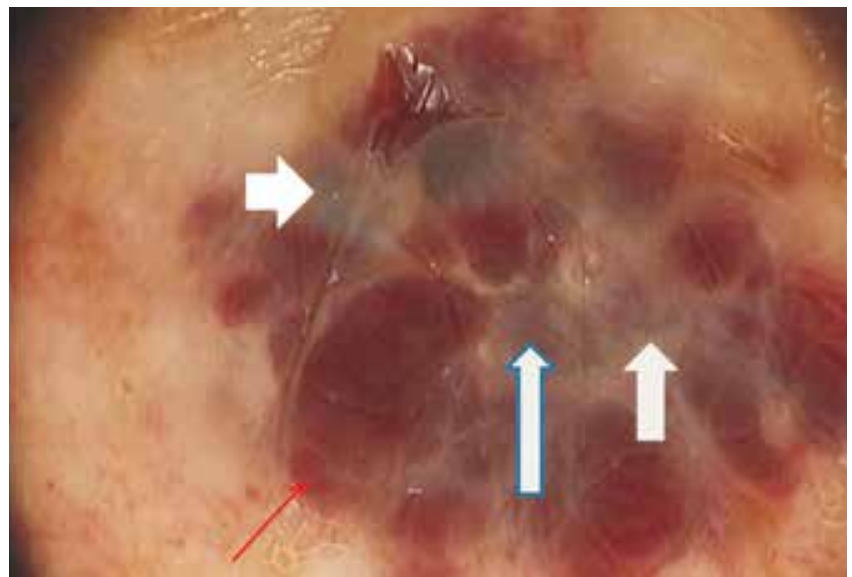

Fig 6 - Dermatoscopia de Angioqueratoma: lacunas mais escuras (seta fina vermelha) que o angioma e véu esbranquiçado (setas largas brancas). 


\section{Caso Clínico}

No que diz respeito ao tratamento, várias opções têm sido propostas com resultados variáveis ${ }^{9-11}$ A maioria dos autores é consensual quanto à necessidade de avaliar a extensão em profundidade do LCN antes de avançar para qualquer tratamento, considerando-se a $R M N^{12}$ a técnica imagiológica ideal.

No caso do nosso doente, a recidiva após uma intervenção prévia e a existência de uma massa retroperitoneal mal definida associada, condicionaram a adoção de uma atitude expectante, com controlo semestral e vigilância das possíveis complicações (linforragia, ulceração e infeção secundária) ${ }^{3}$.

Partilhamos este caso clínico de LCN, essencialmente, pelos aspetos interessantes dermatoscópicos que apresenta, e pela sua difícil abordagem terapêutica.

\section{BIBLIOGRAFIA}

1. Guiote MV, Moreno A, Vieira R, Tellechea $O$, Naranjo $R$, Figueiredo A. Linfangioma circunscrito neviforme. Actas Dermosifiliogr. 2006;97(7):477-8.

2. Martínez Menchón T, Mahiques Santos L, Febrer-Bosch I, Valcuende Cavero F, Fortea Baixauli JM. Lymphangioma circumscriptum: na example of Whimster's hypotesis. Pediatr Dermatol. 2004;21:652-4.

3. Boon L, Vikkula M. Vascular Malformations.In: Wolff K, Johnson RA, editors. Fitzpatrick's Color
Atlas And Synopsis Of Clinical Dermatology. Berkshire: McGraw Hill; 2008.p. 1651-66.

4. Arpia N, Cassano N, Vena GA. Dermoscopic features of cutaneous lymphangioma circumscriptum. Dermatol Surg. 2006; 32(6): 852-4.

5. Wolf IH . Dermoscopic diagnostic of vascular lesions. Clin Dermatol. 2002;20:273-5.

6. Schiller $\mathrm{Pl}$, Itin $\mathrm{PH}$. Angiokeratomas: an up date. Dermatology. 1996; 193:275-82.

7. Goldman L, Gibson SH, Richfield DF. Thrombotic angiokeratoma circunscriptum simulating melanoma. Arch Dermatol. $1981 ; 117: 138-9$.

8. Zaballos P, Daufí C, Puig S, Argenziano G, Moreno-Ramírez D, Cabo H, et al. Dermoscopy of solitary angiokeratoma: a morphological study . Arch Dermatol. 2007; 143:318-2.

9. Bikowski JB, Dumont AM. Lymphangioma circumscritum: treatment with hypertonic saline sclerotherapy. J Am Acad Dermatol. 2005;53:442-4.

10. Huilgol SC, Neill S, Barlow RJ. CO2 laser therapy of vulval lymphangiectasia and lymphamgioma circumscriptum. Dermatol Surg. 2002;28:575-7.

11. Browse NL, Whimster I, Stewart G, Helm CW, Wood JJ. Surgical management of lymphangioma circumscriptum. Br J Surg. 1996;73:585-8.

12. McAlvany JP, Jorizzo JL, Zanolli $D$, Auringer S, Prichard E, Krowchuuk DP, et al. Magnetic resonance imaging of lymphangioma circumscritum. Arch Dermatol. 1993;129:194-7. 